\title{
Front Matter: Volume 8961
}

, "Front Matter: Volume 8961," Proc. SPIE 8961, Fiber Lasers XI: Technology, Systems, and Applications, 896101 (26 March 2014); doi:

10.1117/12.2062794

SPIE. Event: SPIE LASE, 2014, San Francisco, California, United States 


\title{
PROCEEDINGS OF SPIE
}

\section{Fiber Lasers XI: Technology, Systems, and Applications}

\section{Siddharth Ramachandran}

Editor

\author{
3-6 February 2014 \\ San Francisco, California, United States
}

Sponsored by

SPIE

Cosponsored by

NKT Photonics A/S (Denmark)

PolarOnyx, Inc. (United States)

Published by

SPIE 
The papers included in this volume were part of the technical conference cited on the cover and title page. Papers were selected and subject to review by the editors and conference program committee. Some conference presentations may not be available for publication. The papers published in these proceedings reflect the work and thoughts of the authors and are published herein as submitted. The publisher is not responsible for the validity of the information or for any outcomes resulting from reliance thereon.

Please use the following format to cite material from this book:

Author(s), "Title of Paper," in Fiber Lasers XI: Technology, Systems, and Applications, edited by Siddharth Ramachandran, Proceedings of SPIE Vol. 8961 (SPIE, Bellingham, WA, 2014) Article CID Number.

ISSN: 0277-786X

ISBN: 9780819498748

Published by

SPIE

P.O. Box 10, Bellingham, Washington 98227-0010 USA

Telephone +1 3606763290 (Pacific Time) · Fax +1 3606471445

SPIE.org

Copyright (@ 2014, Society of Photo-Optical Instrumentation Engineers.

Copying of material in this book for internal or personal use, or for the internal or personal use of specific clients, beyond the fair use provisions granted by the U.S. Copyright Law is authorized by SPIE subject to payment of copying fees. The Transactional Reporting Service base fee for this volume is $\$ 18.00$ per article (or portion thereof), which should be paid directly to the Copyright Clearance Center (CCC), 222 Rosewood Drive, Danvers, MA 01923. Payment may also be made electronically through CCC Online at copyright.com. Other copying for republication, resale, advertising or promotion, or any form of systematic or multiple reproduction of any material in this book is prohibited except with permission in writing from the publisher. The CCC fee code is 0277-786X/14/\$18.00.

Printed in the United States of America.

Publication of record for individual papers is online in the SPIE Digital Library.

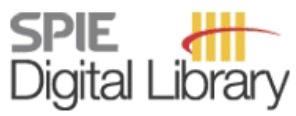

SPIEDigitallibrary.org

Paper Numbering: Proceedings of SPIE follow an e-First publication model, with papers published first online and then in print and on CD-ROM. Papers are published as they are submitted and meet publication criteria. A unique, consistent, permanent citation identifier (CID) number is assigned to each article at the time of the first publication. Utilization of CIDs allows articles to be fully citable as soon as they are published online, and connects the same identifier to all online, print, and electronic versions of the publication. SPIE uses a six-digit CID article numbering system in which:

- The first four digits correspond to the SPIE volume number.

- The last two digits indicate publication order within the volume using a Base 36 numbering

system employing both numerals and letters. These two-number sets start with 00, 01, 02, 03, 04, $05,06,07,08,09,0 A, 0 B \ldots$. 0Z, followed by 10-1Z, 20-2Z, etc.

The CID Number appears on each page of the manuscript. The complete citation is used on the first page, and an abbreviated version on subsequent pages. Numbers in the index correspond to the last two digits of the six-digit CID Number. 


\section{Contents}

$X V$

Conference Committee

NARROW LINEWIDTH

896102 Using a linearly chirped seed suppresses SBS in high-power fiber amplifiers, allows coherent combination, and enables long delivery fibers (Invited Paper) [8961-1] J. O. White, E. Petersen, U.S. Army Research Lab. (United States); J. Edgecumbe, Nufern (United States); G. Rakuljic, N. Satyan, Telaris, Inc. (United States); A. Vasilyev, A. Yariv, California Institute of Technology (United States)

896103 Single-frequency Yb-doped photonic crystal fiber amplifier with 800W output power [8961-2]

C. Robin, I. Dajani, B. Pulford, C. Vergien, Air Force Research Lab. (United States)

896104 Characterization of photonic bandgap fiber for high-power narrow-linewidth optical transport [8961-3]

C. R. Bennett, D. C. Jones, M. A. Smith, A. M. Scott, QinetiQ Ltd. (United Kingdom);

J. K. Lyngsoe, C. Jakobsen, NKT Photonics A/S (Denmark)

896105 Single-frequency 1178nm SDL/Yb-PBGF MOPA with an output power of 31 W [8961-4] T. Leinonen, Tampere Univ. of Technology (Finland); M. Chen, X. Fan, The Univ. of ElectroCommunications (Japan); E. Kantola, Tampere Univ. of Technology (Finland);

A. Shirakawa, The Univ. of Electro-Communications (Japan); M. Guina, Tampere Univ. of Technology (Finland)

\section{BEAM COMBINATION}

896106 Approaching TW-peak powers at $>10 \mathrm{kHz}$ repetition rate by multi-dimensional coherent combining of femtosecond fiber lasers [8961-5]

S. Breitkopf, Friedrich-Schiller-Univ. Jena (Germany); T. Eidam, Friedrich-Schiller-Univ. Jena (Germany) and Helmholtz-Institut Jena (Germany); L. von Grafenstein, Friedrich-SchillerUniv. Jena (Germany); A. Klenke, Friedrich-Schiller-Univ. Jena (Germany) and HelmholtzInstitut Jena (Germany); H. Carstens, S. Holzberger, I. Pupeza, E. Fill, Max-Planck-Institut für Quantenoptik (Germany) and Ludwig-Maximilians-Univ. München (Germany); T. Schreiber, Fraunhofer-Institut für Angewandte Optik und Feinmechanik (Germany); J. Limpert, Friedrich-Schiller-Univ. Jena (Germany), Helmholtz-Institut Jena (Germany), and Fraunhofer Institute for Applied Optics and Precision Engineering (Germany); F. Krausz, Max-PlanckInstitut für Quantenoptik (Germany) and Ludwig-Maximilians-Univ. München (Germany); A. Tünnermann, Friedrich-Schiller-Univ. Jena (Germany), Helmholtz-Institut Jena (Germany), and Fraunhofer Institute for Applied Optics and Precision Engineering (Germany)

896107 Coherent beam combining performance in harsh environment [8961-6]

L. Lombard, G. Canat, A. Durecu, P. Bourdon, ONERA (France) 
8961 OC High-power narrowline 1.5- $\mathrm{mm}$ fiber amplifier lidar transmitter for atmospheric $\mathrm{CO}_{2}$ detection [8961-11]

W. Lu, D. Engin, M. Storm, S. Gupta, Fibertek, Inc. (United States)

8961 OD High power fiber lasers in geothermal, oil and gas (Invited Paper) [8961-12]

M. S. Zediker, Foro Energy, Inc. (United States)

\section{BANDGAP AND MICROSTRUCTURED FIBERS}

8961 OF Design of double-cladding large mode area all-solid photonic bandgap fibers [8961-14]

E. Coscelli, Univ. degli Studi di Parma (Italy); T. T. Alkeskjold, NKT Photonics A/S (Denmark);

A. Cucinotta, S. Selleri, Univ. degli Studi di Parma (Italy)

8961 OG Novel multifocus tomography for measurement of microstructured and multicore optical fibers [8961-15]

A. D. Yablon, Interfiber Analysis (United States)

8961 Ol Negative curvature hollow core fibers: design, fabrication, and applications (Invited Paper) [8961-17]

A. D. Pryamikov, Fiber Optics Research Ctr. (Russian Federation)

\section{ULTRASHORT PULSE LASERS I}

8961 OK Towards high power and high energy femtosecond fiber lasers [8961-19]

P. Wan, L.-M. Yang, J. Liu, PolarOnyx, Inc. (United States)

8961 OL Divided-pulse nonlinear compression [8961-20]

F. Guichard, Amplitude Systèmes (France) and Lab. Charles Fabry, Institut d'Optique, CNRS, Univ. Paris-Sud (France); Y. Zaouter, Amplitude Systèmes (France); M. Hanna, F. Druon, Lab. Charles Fabry, Institut d'Optique, CNRS, Univ. Paris-Sud (France); E. Mottay, Amplitude Systèmes (France); P. Georges, Lab. Charles Fabry, Institut d'Optique, CNRS, Univ. Paris-Sud (France)

8961 OM Analysis of divided-pulse amplification for high-energy extraction [8961-21] M. Kienel, A. Klenke, S. Hädrich, T. Eidam, Friedrich-Schiller-Univ. Jena (Germany) and Helmholtz-Instituł Jena (Germany); J. Limpert, A. Tünnermann, Friedrich-Schiller-Univ. Jena (Germany), Fraunhofer-Institut für Angewandte Optik und Feinmechanik (Germany), and Helmholtz-Institut Jena (Germany)

$89610 \mathrm{~N} 215 \mu \mathrm{J} 16 \mathrm{~W}$ femtosecond fiber laser for precision industrial micro-machining [8961-22] K. Kim, X. Peng, W. Lee, Raydiance, Inc. (United States); X. GU, JDSU (United States); M. Mielke, Raydiance, Inc. (United States) 
8961 OS Fibers design with a bend-compensated cladding for distributed wavelength filtering [8961-27]

J. M. Fini, J. W. Nicholson, OFS Labs. (United States)

8961 OT $\mathrm{Yb}^{3+}$ doped ribbon fiber for high-average power lasers and amplifiers [8961-28]

D. R. Drachenberg, M. J. Messerly, P. H. Pax, A. K. Sridharan, J. B. Tassano, J. W. Dawson, Lawrence Livermore National Lab. (United States)

8961 OU Low-loss hybrid fiber with zero dispersion wavelength shifted to $1 \boldsymbol{\mu m}$ [8961-29]

S. S. Aleshkina, M. E. Likhachev, A. K. Senatorov, M. M. Bubnov, Fiber Optics Research Ctr. (Russian Federation); M. Yu. Salaganskii, A. N. Guryanov, Institute of Chemistry of High-Purity Substances (Russian Federation)

\section{HIGH POWER I}

8961 OV 3kW single-mode direct diode-pumped fiber laser [8961-30]

V. Khitrov, J. D. Minelly, R. Tumminelli, V. Petit, E. S. Pooler, Coherent, Inc. (United States)

$8961 \mathrm{OW} \quad 146 \mathrm{~W}$ continuous wave ytterbium doped fiber amplifier at $1009 \mathrm{~nm}$ [8961-31]

F. Beier, Friedrich-Schiller-Univ. Jena (Germany) and Fraunhofer-Institut für Angewandte Optik und Feinmechanik (Germany); H.-J. Otto, Friedrich-Schiller-Univ. Jena (Germany); B. Sattler, M. Ploetner, N. Haarlammert, O. de Vries, Fraunhofer-Institut für Angewandte Optik und Feinmechanik (Germany); C. Jauregui, Friedrich-Schiller-Univ. Jena (Germany); T. Schreiber, Fraunhofer-Institut für Angewandte Optik und Feinmechanik (Germany); J. Limpert, Friedrich-Schiller-Univ. Jena (Germany) and Fraunhofer-Institut für Angewandte Optik und Feinmechanik (Germany); R. Eberhardt, Fraunhofer-Institut für Angewandte Optik und Feinmechanik (Germany); A. Tünnermann, Friedrich-Schiller-Univ. Jena (Germany) and Fraunhofer-Institut für Angewandte Optik und Feinmechanik (Germany)

8961 OX Yb-free Er-doped all-fiber amplifier cladding-pumped at $976 \mathrm{~nm}$ with output power in excess of $100 \mathrm{~W}$ [8961-32]

L. V. Kotov, Fiber Optics Research Ctr. (Russian Federation) and Moscow Institute of Physics and Technology (Russian Federation); M. E. Likhachev, M. M. Bubnov, O. I. Medvedkov, Fiber Optics Research Ctr. (Russian Federation); M. V. Yashkov, A. N. Guryanov, Institute of Chemistry of High-Purity Substances (Russian Federation); S. Février, XLIM, CNRS, Univ. de Limoges (France); J. Lhermite, E. Cormier, CeLIA Lab., CNRS, Univ. Bordeaux 1 (France)

$89610 Z$ Investigation of a large core $976 \mathrm{~nm} \mathrm{Yb}$ fiber laser for high brightness fiber-based pump sources [8961-34]

M. Leich, M. Jäger, S. Grimm, J. Dellith, D. Hoh, S. Jetschke, M. Becker, A. Hartung, T. Eschrich, J. Kobelke, H. Bartelt, Leibniz Institute of Photonic Technology e.V. (Germany) 
896110 Bursting for enhanced ablation of materials (Invited Paper) [8961-35]

S. Hendow, Adaptive Laser Processing (United States); E. Rea, Coherent, Inc. (United States); N. Kosa, Adaptive Laser Processing (United States); M. Bengtsson, Coherent, Inc. (United States); S. Shakir, Tau Technologies (United States)

896111 High peak- and average-power pulse shaped fiber laser in the ns-regime applying stepindex XLMA gain fibers [8961-36]

R. Dinger, F.-P. Grundmann, C. Hapke, S. Ruppik, ROFIN-SINAR Laser GmbH (Germany)

896112 High-peak power, flexible-pulse parameter, chirally coupled core (3C) fiber-based picosecond MOPA systems [8961-37]

T. S. McComb, D. McCal, R. Farrow, T. L. Lowder, D. Logan, J. Green, T. N. Kutscha, C. Ye, V. Aallos, J. J. Koponen, G. Fanning, nLIGHT Corp. (United States)

896113 An all-fiber high-energy cladding-pumped 93 nanosecond Q-switched fiber laser using an $\mathrm{Y}^{3+}$-doped fiber saturable absorber [8961-38]

S. W. Moore, B. D. Patterson, D. B. S. Soh, S. E. Bisson, Sandia National Labs. (United States)

896114 High-power monolithic fiber amplifiers based on advanced photonic crystal fiber designs [8961-39]

D. L. Sipes Jr., J. D. Tafoya, D. S. Schulz, Optical Engines, Inc. (United States); T. T. Alkeskjold, J. Weirich, C. B. T. Olausson, NKT Photonics A/S (Denmark)

\section{MATERIALS AND FABRICATION}

896115 Plasma outside deposition (POD) of fluorine doped silica for high-power laser applications (Invited Paper) [8961-40]

A. Langner, G. Schötz, Heraeus Quarzglas GmbH \& Co. KG (Germany)

896116 The Yb-doped aluminosilicate fibers photodarkening mechanism based on the chargetransfer state excitation [8961-41]

A. A. Rybaltovsky, K. K. Bobkov, V. V. Velmiskin, Fiber Optics Research Ctr. (Russian Federation); A. A. Umnikov, Institute of Chemistry of High-Purity Substances (Russian Federation); I. A. Shestakova, POLYUS Research and Development Institute (Russian Federation); A. N. Guryanov, Institute of Chemistry of High-Purity Substances (Russian Federation); M. E. Likhachev, M. M. Bubnov, E. M. Dianov, Fiber Optics Research Ctr. (Russian Federation)

896118 Fabrication and characterization of a phosphosilicate YDF with high Yb absorbance and low background loss [8961-43]

S.-J. Kim, Y. Hujimaki, H. Taniguchi, H. Kinoshita, K. Sato, Mitsubishi Cable Industries, Ltd. (Japan) 
MID-IR SOURCES

8961 1A Development of high-power holmium-doped fibre amplifiers [8961-45]

A. Hemming, N. Simakov, A. Davidson, M. Oermann, L. Corena, D. Stepanov, N. Carmody, J. Haub, Defence Science and Technology Organisation (Australia); R. Swain, Sub-Micron Engineering Corp. (United States); A. Carter, Nufern (United States)

8961 1B Efficiency improvement in Thulium-doped fibers via excited state pumping [8961-46] C. Jauregui, F. Stutzki, F. Jansen, Friedrich-Schiller-Univ. Jena (Germany); J. Limpert, Friedrich-Schiller-Univ. Jena (Germany) and Helmholtz-Institut Jena (Germany); A. Tünnermann, Fraunhofer-Institut für Angewandte Optik und Feinmechanik (Germany), Helmholtz-Institut Jena (Germany), and Fraunhofer-Institut für Angewandte Optik und Feinmechanik (Germany)

8961 1C All-fiber optical supercontinuum sources in 1.7-3.2 $\mu \mathrm{m}$ range [8961-47] V. V. Dvoyrin, I. T. Sorokina, Norwegian Univ. of Science and Technology (Norway)

\section{ULTRASHORT PULSE LASER II}

8961 ID 2.1mJ, 210W femtosecond fiber CPA system [8961-48]

A. Klenke, Friedrich-Schiller-Univ. Jena (Germany) and Helmholtz-Institut Jena (Germany); A. Hoffmann, Friedrich-Schiller-Univ. Jena (Germany); S. Hädrich, Friedrich-Schiller-Univ. Jena (Germany) and Helmholtz-Institut Jena (Germany); T. Eidam, Friedrich-Schiller-Univ. Jena (Germany) and Fraunhofer-Institut für Angewandte Optik und Feinmechanik (Germany); T. Gottschall, Friedrich-Schiller-Univ. Jena (Germany); J. Limpert,

A. Tünnermann, Friedrich-Schiller-Univ. Jena (Germany), Fraunhofer-Institut für Angewandte Optik und Feinmechanik (Germany), and Helmholtz-Institut Jena (Germany)

8961 IE High-pulse energy and average-power ultrashort laser pulses via nonlinear compression of coherently combined fiber CPA system [8961-49]

S. Hädrich, A. Klenke, Friedrich-Schiller-Univ. Jena (Germany) and Helmholtz-Institut Jena (Germany); A. Hoffmann, T. Eidam, T. Gottschall, Friedrich-Schiller-Univ. Jena (Germany); J. Rothhardt, J. Limpert, Friedrich-Schiller-Univ. Jena (Germany) and Helmholtz-Institut Jena (Germany); A. Tünnermann, Friedrich-Schiller-Univ. Jena (Germany), Helmholtz-Institut Jena (Germany), and Fraunhofer-Institut für Angewandte Optik und Feinmechanik (Germany)

$8961 \mathrm{lF}$ High average power and energetic femtosecond fiber laser using chirped-and dividedpulse amplification [8961-50]

Y. Zaouter, Amplitude Systèmes (France); F. Guichard, Amplitude Systèmes (France) and Lab. Charles Fabry, Institut d'Optique, CNRS, Univ. Paris-Sud (France); M. Hanna, Lab. Charles Fabry, Institut d'Optique, CNRS, Univ. Paris-Sud (France); F. Morin, C. Hönninger, Amplitude Systèmes (France); F. Druon, Lab. Charles Fabry, Institut d'Optique, CNRS, Univ. Paris-Sud (France); E. Mottay, Amplitude Systèmes (France); P. Georges, Lab. Charles Fabry, Institut d'Optique, CNRS, Univ. Paris-Sud (France) 
8961 IG Spectral synthesis to overcome gain-narrowing in femtosecond fiber amplifiers [8961-51] F. Guichard, Amplitude Systèmes (France) and Lab. Charles Fabry, Institut d'Optique, CNRS, Univ. Paris-Sud (France); M. Hanna, Lab. Charles Fabry, Institut d'Optique, CNRS, Univ. Paris-Sud (France); L. Lombard, ONERA (France); Y. Zaouter, Amplitude Systèmes (France); F. Druon, Lab. Charles Fabry, Institut d'Optique, CNRS, Univ. Paris-Sud (France); E. Mottay, Amplitude Systèmes (France); P. Georges, Lab. Charles Fabry, Institut d'Optique, CNRS, Univ. Paris-Sud (France)

\section{NOVEL FIBERS AND DESIGNS II}

8961 1K High gain ytterbium doped Ge pedestal large pitch fiber [8961-55]

C. Gaida, F. Stutzki, F. Jansen, H.-J. Otto, T. Eidam, C. Jauregui, Friedrich-Schiller-Univ. Jena (Germany); J. Limpert, Friedrich-Schiller-Univ. Jena (Germany) and Helmholtz-Institut Jena (Germany); A. Tünnermann, Friedrich-Schiller-Univ. Jena (Germany), Helmholtz-Institut Jena (Germany), and Fraunhofer-Institut für Angewandte Optik und Feinmechanik (Germany)

8961 1L Double-clad large mode area Er-doped fiber for high-energy and high-peak power amplifiers [8961-56]

L. V. Kotov, Fiber Optics Research Ctr. (Russian Federation) and Moscow Institute of Physics and Technology (Russian Federation); M. M. Bubnov, Fiber Optics Research Ctr. (Russian Federation); D. S. Lipatov, Moscow Institute of Physics and Technology (Russian Federation); A. N. Guryanov, Institute of Chemistry of High-Purity Substances (Russian Federation); S. Février, XLIM, CNRS, Univ. de Limoges (France); J. Lhermite, E. Cormier, CelIA Lab., CNRS, Univ. Bordeaux 1 (France); M. Yu. Koptev, E. A. Anashkina,

S. V. Muraviev, A. V. Andrianov, A. V. Kim, Institute of Applied Physics (Russian Federation); M. E. Likhachev, Fiber Optics Research Ctr. (Russian Federation)

8961 1M Single mode fibers with antireflective surface structures for high power laser applications [8961-57]

L. E. Busse, U.S. Naval Research Lab. (United States); C. M. Florea, Sotera Defense Solutions, Inc. (United States); L. B. Shaw, U.S. Naval Research Lab. (United States); I. D. Aggarwal, Sotera Defense Solutions, Inc. (United States); J. S. Sanghera, U.S. Naval Research Lab. (United States)

$8961 \mathrm{lN} \quad$ Breaking the symmetry for enhanced higher-order mode delocalization [8961-58] F. Stutzki, F. Jansen, C. Jauregui, Friedrich-Schiller-Univ. Jena (Germany); J. Limpert, Friedrich-Schiller-Univ. Jena (Germany) and Helmholtz-Institut Jena (Germany);

A. Tünnermann, Fraunhofer-Institut für Angewandte Optik und Feinmechanik (Germany), Helmholtz-Institut Jena (Germany), and Fraunhofer-Institut für Angewandte Optik und Feinmechanik (Germany)

\section{FIBER PUMPED FREQUENCY CONVERSION}

8961 iP A scalable high-power yellow laser source based on frequency doubling of a combined Yb-Raman fiber amplifier [8961-60]

E. E. Rowen, G. Vashdi, J. Lasri, E. Inbar, V-Gen Ltd. (Israel) 
8961 IR Threshold power and fiber degradation induced modal instabilities in high-power fiber amplifiers based on large mode area fibers [8961-62]

K. Brar, M. Savage-Leuchs, J. Henrie, S. Courtney, C. Dilley, R. Afzal, E. Honea, Lockheed Martin Laser and Sensor Systems (United States)

8961 is Raising the mode instability thresholds of fiber amplifiers [8961-63]

A. V. Smith, J. J. Smith, AS-Photonics, LLC (United States)

8961 1T Analysis of stimulated Raman scattering in cw kW fiber oscillators [8961-64]

T. Schreiber, A. Liem, E. Freier, C. Matzdorf, R. Eberhardt, Fraunhofer-Institut für Angewandte Optik und Feinmechanik (Germany); C. Jauregui, J. Limpert, Friedrich-Schiller-Univ. Jena (Germany); A. Tünnermann, Fraunhofer-Institut für Angewandte Optik und Feinmechanik (Germany) and Friedrich-Schiller-Univ. Jena (Germany)

$89611 \mathrm{U}$ Numerical analysis of modal instability onset in fiber amplifiers [8961-65]

B. G. Ward, U.S. Air Force Academy (United States)

\section{POSTER SESSION}

8961 IV High-brightness fiber-coupled pump modules in fiber laser applications [8961-66] D. Hemenway, W. Urbanek, K. Hoener, K. Kennedy, L. Bao, D. Dawson, E. Cragerud, D. Balsley, G. Burkholder, M. Reynolds, K. Price, J. Haden, M. Kanskar, D. Kliner, nLIGHT Corp. (United States)

8961 IW Experimental study of SBS suppression via white noise phase modulation [8961-67] B. Anderson, CREOL, The College of Optics and Photonics, Univ. of Central Florida (United States); C. Robin, A. Flores, I. Dajani, Air Force Research Lab. (United States)

8961 1X High energy, high average and peak power phosphate-glass fiber amplifiers for $1 \mathrm{micron}$ band [8961-68]

M. Akbulut, A. Miller, K. Wiersma, J. Zong, D. Rhonehouse, D. Nguyen, A. Chavez-Pirson, NP Photonics, Inc. (United States)

8961 IY Coherent combining of SHG converters through active phase control of the fundamental waves [8961-69]

A. Durécu, C. Aubert, G. Canat, J. Le Gouët, L. Lombard, P. Bourdon, ONERA (France)

896120 Ho-doped fiber for high energy laser applications [8961-71]

E. J. Friebele, C. G. Askins, U.S. Naval Research Lab. (United States); J. R. Peele, Sotera Defense Solutions, Inc. (United States); B. M. Wright, U.S. Naval Research Lab. (United States); N. J. Condon, Digital Optics Technologies, Inc. (United States); S. O'Connor, Naval Air Warfare Ctr. Aircraft Div. (United States); C. G. Brown, Sotera Defense Solutions, Inc. (United States); S. R. Bowman, U.S. Naval Research Lab. (United States) 
$896121 \quad$ Effective mitigation of photodarkening in Yb-doped lasers based on Al-silicate using UV/visible light [8961-72]

R. Piccoli, Swansea Univ. (United Kingdom); T. Robin, ilXFiber SAS (France); D. Méchin, PERFOS (France); T. Brand, DILAS Diodenlaser GmbH (Germany); U. Klotzbach, IWS

Fraunhofer (Germany); S. Taccheo, Swansea Univ. (United Kingdom)

896122 All-fiber Raman oscillator for the generation of radially and azimuthally polarized beams [8961-73]

C. Jocher, C. Jauregui, Friedrich-Schiller-Univ. Jena (Germany); M. Becker, M. Rothhardt, Institut für Photonische Technologien e.V. (Germany); J. Limpert, A. Tünnermann, Friedrich-Schiller-Univ. Jena (Germany) and Fraunhofer-Institut für Angewandte Optik und Feinmechanik (Germany

896123 Optimization of laser fibers for high pump light absorption [8961-74]

J. Bierlich, J. Kobelke, S. Jetschke, S. Grimm, S. Unger, K. Schuster, IPHT Jena (Germany)

896124 Completely monolithic linearly polarized high-power fiber laser oscillator [8961-75] S. Belke, F. Becker, B. Neumann, S. Ruppik, U. Hefter, ROFIN-SINAR Laser GmbH (Germany)

896126 Single-crystal rare-earth doped YAG fiber lasers grown by the laser-heated pedestal growth technique [8961-77]

C. D. Nie, J. A. Harrington, Rutgers, The State Univ. of New Jersey (United States); Y. Li, E. G. Johnson, Clemson Univ. (United States); E. F. Cloos, S. C. Rand, P. Machado, Univ. of Michigan (United States); R. K. Shori, SPAWAR Systems Ctr. (United States)

896128 Passive mitigation of mode instabilities [8961-79]

C. Jauregui, H.-J. Otto, F. Stutzki, F. Jansen, J. Limpert, Friedrich-Schiller-Univ. Jena (Germany); A. Tünnermann, Friedrich-Schiller-Univ. Jena (Germany), Helmholtz-Institut Jena (Germany), and Fraunhofer-Institut für Angewandte Optik und Feinmechanik (Germany)

896129 Fabrication of microstructured fibers from preforms with sealed top-end holes [8961-80] S. L. Semjonov, A. N. Denisov, A. K. Senatorov, Fiber Optics Research Ctr. (Russian Federation)

8961 2A Dual-wavelength fiber mode-locked laser based on graphene saturable absorber [8961-81]

J. Sotor, G. Soboń, Wroclaw Univ. of Technology (Poland); I. Pasternak, Institute of Electronic Materials Technology (Poland); K. Krzempek, G. Dudzik, Wroclaw Univ. of Technology (Poland); A. Krajewska, Institute of Electronic Materials Technology (Poland) and Military Univ. of Technology (Poland); W. Strupinski, Institute of Electronic Materials Technology (Poland); K. M. Abramski, Wroclaw Univ. of Technology (Poland)

8961 2B The effect of polarization in passive coherent beam combining of fiber lasers [8961-82] H.-S. Chiang, J. R. Leger, Univ. of Minnesota (United States); E. Huszar, Bethlen Gábor Református Gimnázium (Hungary); J. Nilsson, J. Sahu, Univ. of Southampton (United Kingdom)

8961 2F 980-nm random fiber laser directly pumped by a high-power 938-nm laser diode [8961-86] S. A. Babin, E. I. Dontsova, S. I. Kablukov, Institute of Automation and Electrometry (Russian Federation) 
$89612 \mathrm{H} \quad 16 \mathrm{~W}$ pulsed green laser based on efficient frequency conversion of an $\mathrm{Yb}$-doped fibre laser externally modulated by a semiconductor optical amplifier [8961-88]

A. Haboucha, CNRS Foton, Univ. de Rennes 1 (Canada); A. Mugnier, Quantel (France); C. Pareige, A. Fernandez, T. Chartier, CNRS Foton, Univ. de Rennes 1 (France); D. Pureur, Quantel (France)

896121 Spatially resolved 3D measurements of long-period gratings written by fs-laser inscription in large mode area fibers [8961-89]

A. Kliner, Fraunhofer-Institut für Angewandte Optik und Feinmechanik (Germany) and Friedrich-Schiller-Univ. Jena (Germany); R. G. Krämer, C. Voigtländer, Friedrich-Schiller-Univ. Jena (Germany); F. Theuer, T. Schreiber, R. Eberhardt, Fraunhofer-Institut für Angewandte Optik und Feinmechanik (Germany); S. Nolte, A. Tünnermann, Fraunhofer-Institut für Angewandte Optik und Feinmechanik (Germany) and Friedrich-Schiller-Univ. Jena (Germany)

8961 2J Ultra-low noise optical phase-locked loop [8961-90]

S. Ayotte, A. Babin, F. Costin, TeraXion Inc. (Canada)

$89612 \mathrm{~K}$ Performance of $\mathrm{kW}$ class fiber amplifiers spanning a broad range of wavelengths: 1028 1100nm [8961-91]

Y. Huang, J. Edgecumbe, J. Ding, R. Holten, P. Ahmadi, C.-H. Wang, C. Guintrand,

K. Farley, S. Christensen, K. Tankala, Nufern (United States)

$89612 \mathrm{~L} \quad$ Power scaling through narrowband ASE seeding in pulsed MOPA fiber systems [8961-92] M. Melo, Univ. of Porto (Portugal) and MWTechnologies, Ltd. (Portugal); J. M. Sousa, Datalogic, S.p.A. (Italy)

$89612 \mathrm{M}$ Development of narrow-linewidth $\mathrm{Yb}$ - and Er-fiber lasers and frequency mixing for ArF excimer laser seeding [8961-93]

H. Xuan, Z. Zhao, The Univ. of Tokyo (Japan); H. Igarashi, S. Ito, K. Kakizaki, Gigaphoton Inc. (Japan); Y. Kobayashi, The Univ. of Tokyo (Japan)

$89612 \mathrm{~N}$ Polarization maintaining, high-power and high-efficiency $(6+1) \times 1$ pump/signal combiner [8961-94]

V. I. Kopp, J. Park, M. Wlodawski, J. Singer, D. Neugroschl, Chiral Photonics, Inc. (United States)

$89612 \mathrm{P}$ Fiber amplifiers under thermal loads leading to transverse mode instability [8961-96] M. M. Johansen, K. R. Hansen, DTU Fotonik (Denmark); T. T. Alkeskjold, NKT Photonics A/S (Denmark); J. Laegsgaard, DTU Fotonik (Denmark)

$89612 \mathrm{Q}$ Thermo-optical effects in Tm-doped large mode area photonic crystal fibers [8961-97] C. Molardi, E. Coscelli, A. Cucinotta, S. Selleri, Univ. degli Studi di Parma (Italy)

896125 Intense supercontinuum generation in a nanosecond nonlinear all-PM-fiber power amplifier [8961-99]

C.-L. Chang, National Taiwan Univ. (Taiwan); Y.-Y. Lin, National Tsing Hua Univ. (Taiwan); P.-Y. Lai, National Central Univ. (Taiwan); Y.-Y. Li, D.-Y. Jheng, National Taiwan Univ. (Taiwan); S.-H. Chen, National Central Univ. (Taiwan); S.-L. Huang, National Taiwan Univ. (Taiwan) 
$89612 \mathrm{~T} \quad$ Effective suppression of stimulated Raman scattering in high power fiber amplifiers using double-pass scheme [8961-100]

P.-Y. Lai, National Central Univ. (Taiwan); C.-L. Chang, S.-L. Huang, National Taiwan Univ. (Taiwan); S.-H. Chen, National Central Univ. (Taiwan)

$89612 U$ Optical fiber designs for beam shaping [8961-101]

K. Farley, M. Conroy, C.-H. Wang, J. Abramczyk, Nufern (United States); S. Campbell, Optoskand AB (Sweden); G. Oulundsen, K. Tankala, Nufern (United States)

$89612 \mathrm{~V}$ Adjustment of double resonance in short cavity Brillouin fiber lasers [8961-102]

C. A. López-Mercado, V. V. Spirin, Ctr. de Investigación Científica y de Educación Superior de Ensenada (Mexico); S. I. Kablukov, E. A. Zlobina, Institute of Automation and Electrometry (Russian Federation); I. O. Zolotovsky, Ulyanovsk State Univ. (Russian Federation); P. Mégret, Univ. de Mons (Belgium); A. A. Fotiadi, Ulyanovsk State Univ. (Russian Federation), Univ. de Mons (Belgium), and loffe Physico-Technical Institute (Russian Federation)

$89612 \mathrm{~W} \quad$ Mode coupling in few-mode large-mode-area fibers [8961-103]

C. Ye, J. Koponen, V. Aallos, L. Petit, O. Kimmelma, T. Kokki, nLIGHT Corp. (Finland)

$89612 \mathrm{X}$ Frequency conversion through spontaneous degenerate four wave mixing in large mode area hybrid photonic crystal fibers [8961-104]

S. R. Petersen, DTU Fotonik (Denmark); T. T. Alkeskjold, C. B. Olausson, NKT Photonics A/S (Denmark); J. Laegsgaard, DTU Fotonik (Denmark)

$89612 Z$ Time-frequency-domain dispersion measurement in rare earth doped large effective mode area multicore fibers [8961-106]

T. Baselt, Ch. Taudt, P. Hartmann, Westsächsische Hochschule Zwickau (Germany)

896132 Optical characterization, luminescence properties of $\mathrm{Er}^{3+}$ and $\mathrm{Er}^{3+} / \mathrm{Yb}^{3+} \mathrm{co}$-doped tellurite glasses for broadband amplification [8961-111]

M. Seshadri, L. C. Barbosa, J. A. P. Ferencz Junior, Univ. Estadual de Campinas (Brazil)

896133 Photonic crystal fiber pump combiner for high-peak power all-fiber thulium lasers [8961-112]

A. Sincore, CREOL, The College of Optics and Photonics, Univ. of Central Florida (United States); J. Tafoya, D. Sipes Jr., Optical Engines, Inc. (United States); L. Leick, NKT Photonics A/S (Denmark); L. Shah, M. Richardson, CREOL, The College of Optics and Photonics, Univ. of Central Florida (United States)

896134 Spectral and temporal phase measurement by optical frequency-domain reflectometry [8961-114]

B. Robillart, Institute Mines-Télécom and Télécom SudParis, CNRS (France); C. Calò, Lab. for Photonics and Nanostructures, CNRS (France); A. Fall, F. Lamare, Y. Gottesman, B.-E. Benkelfat, Institute Mines-Télécom and Télécom SudParis, CNRS (France)

896135 Effect of linewidth enhancement factor in actively mode-locked ring laser [8961-1 15] A. Takada, M. Saika, S. Nagano, Topcon Corp. (Japan) 
896137 Influence of zero dispersion wavelength on supercontinuum generation in near infrared, visible, and UV range for a series of microstructured fibres [8961-117]

Z. Holdynski, M. Napierala, M. Szymanski, M. Murawski, Military Univ. of Technology (Poland) and InPhoTech Ltd. (Poland); P. Mergo, Univ. of Maria Curie-Sklodowska (Poland); P. Marc, L. R. Jaroszewicz, Military Univ. of Technology (Poland); T. Nasilowski, Military Univ. of Technology (Poland) and InPhoTech Ltd. (Poland)

896138 Analysis of supercontinuum generated with endlessly single mode new type of microstructured fibre series with near-visible zero dispersion wavelength [8961-118] Z. Holdynski, M. Napierala, Military Univ. of Technology (Poland) and InPhoTech Ltd. (Poland); U. A. Laudyn, M. A. Karpierz, Warsaw Univ. of Technology (Poland); M. Szymanski, M. Murawski, Military Univ. of Technology (Poland) and InPhoTech Ltd. (Poland); P. Mergo, Univ. of Maria Curie-Sklodowska (Poland); P. Marc, L. R. Jaroszewicz, Military Univ. of Technology (Poland); T. Nasilowski, Military Univ. of Technology (Poland) and InPhoTech Ltd. (Poland)

896139 Spectroscopic properties of $\mathrm{Ho}^{3+}, \mathrm{Tm}^{3+}$, and $\mathrm{Ho}^{3+} / \mathrm{Tm}^{3+}$ doped tellurite glasses for fiber laser applications [8961-119]

M. Seshadri, J. A. P. Ferencz Junior, Univ. Estadual de Campinas (Brazil); Y. C. Ratnakaram, Sri Venateswara Univ. (India); L. C. Barbosa, Univ. Estadual de Campinas (Brazil)

$89613 \mathrm{~A}$ Effect of mode locking technique on the filtering bandwidth limitation in all normal dispersion femtosecond fiber laser [8961-120]

H. E. Kotb, Univ. of Ottawa (Canada); M. A. Abdelalim, Electronics Research Institute (Egypt); H. Anis, Univ. of Ottawa (Canada)

$89613 \mathrm{C}$ Automated image magnification for $\mathrm{CO}_{2}$ laser glass processing system (LZM-100 LazerMaster) [8961-122]

M. E. Harju, H. Sugawara, T. Mizushima, Air Force Research Lab. (United States)

$89613 \mathrm{~F}$ High power fiber amplifier with adjustable repetition rate for use in all-fiber supercontinuum light sources [8961-125]

T. Baselt, Ch. Taudt, P. Hartmann, Westsächsische Hochschule Zwickau (Germany)

$89613 \mathrm{H} \quad$ Stimulated Brillouin scattering suppression with a chirped laser seed: comparison of dynamical model to experimental data [8961-125]

E. Petersen, Z. Yang, J. O. White, U.S. Army Research Lab. (United States); G. Rakuljic, Telaris, Inc. (United States); N. Satyan, A. Vasilyev, A. Yariv, California Institute of Technology (United States)

Author Index 
Proc. of SPIE Vol. $8961896101-14$

Downloaded From: https://www.spiedigitallibrary.org/conference-proceedings-of-spie on 26 Apr 2023 Terms of Use: https://www.spiedigitallibrary.org/terms-of-use 


\title{
Conference Committee
}

\author{
Symposium Chairs
}

Bo Gu, Bos Photonics (United States)

Andreas Tünnermann, Fraunhofer-Institut für Angewandte Optik und Feinmechanik (Germany) and Friedrich-Schiller-Universität Jena (Germany)

Symposium Co-chairs

Guido Hennig, Daetwyler Graphics AG (Swtizerland)

Yongfeng Lu, University of Nebraska-Lincoln (United States)

Program Track Chair

Gregory J. Quarles, Optoelectronics Management Network (United States)

Conference Chair

Siddharth Ramachandran, Boston University (United States)

Conference Co-chair

Brandon Shaw, U.S. Naval Research Laboratory (United States)

Conference Program Committee

Thomas T. Alkeskjold, NKT Photonics A/S (Denmark)

Paulo Almeida, Fianium Ltd. (United Kingdom)

John Ballato, Clemson University (United States)

Adrian L. Carter, Nufern (United States)

Fabio Di Teodoro, The Aerospace Corporation (United States)

Ingmar Hartl, Deutsches Elektronen-Synchrotron (Germany)

Clifford Headley III, OFS Laboratories (United States)

Sami T. Hendow, Adaptive Laser Processing (United States)

Eric C. Honea, Lockheed Martin Aculight (United States)

Jens Limpert, Friedrich-Schiller-Universität Jena (Germany)

Jian Liu, PolarOnyx, Inc. (United States)

John D. Minelly, Coherent, Inc. (United States)

Peter F. Moulton, Q-Peak, Inc. (United States)

Martin H. Muendel, JDSU (United States)

Hans-Jürgen Otto, Friedrich-Schiller-Universität Jena (Germany)

Craig A. Robin, Lockheed Martin Aculight (United States) 
Akira Shirakawa, The University of Electro-Communications (Japan) Daniel B. Soh, Sandia National Laboratories (United States)

Ji Wang, Corning Inc. (United States)

Michalis N. Zervas, University of Southampton (United Kingdom)

\section{Session Chairs}

1 Narrow Linewidth

Akira Shirakawa, The University of Electro-Communications (Japan)

2 Beam Combination

Craig A. Robin, Lockheed Martin Aculight (United States)

3 Applications

Clifford Headley III, OFS Laboratories (United States)

$4 \quad$ Bandgap and Microstructured Fibers

Siddharth Ramachandran, Boston University (United States)

5 Ultrashort Pulse Lasers I

Ingmar Hartl, Deutsches Elektronen-Synchrotron (Germany)

6 Pioneers of Fiber Optics

Siddharth Ramachandran, Boston University (United States)

7 Novel Fibers and Designs

Siddharth Ramachandran, Boston University (United States)

8 High Power I

Sami T. Hendow, Adaptive Laser Processing (United States)

9 High Power II

Eric C. Honea, Lockheed Martin Aculight (United States)

10 Materials and Fabrication

Adrian L. Carter, Nufern (United States)

11 Mid-IR Sources

Brandon Shaw, U.S. Naval Research Laboratory (United States)

12 Ultrashort Pulse Laser II

Jian Liu, PolarOnyx, Inc. (United States)

13 Novel Fibers and Designs II

Ji Wang, Corning Incorporated (United States) 
14 Fiber Pumped Frequency Conversion

Peter F. Moulton, Q-Peak, Inc. (United States)

15 Characterization

John D. Minelly, Coherent, Inc. (United States)

Proc. of SPIE Vol. $8961896101-17$

Downloaded From: https://www.spiedigitallibrary.org/conference-proceedings-of-spie on 26 Apr 2023 Terms of Use: https://www.spiedigitallibrary.org/terms-of-use 
Proc. of SPIE Vol. $8961896101-18$

Downloaded From: https://www.spiedigitallibrary.org/conference-proceedings-of-spie on 26 Apr 2023 Terms of Use: https://www.spiedigitallibrary.org/terms-of-use 